\title{
Time Course of Brainstem Pathophysiology during First Month in Term Infants after Perinatal Asphyxia, Revealed by MLS BAER Latencies and Intervals
}

\author{
ZE D. JIANG, DOROTHEA M. BROSI, JIN WANG, XIU XU, GUO Q. CHEN, XIAO M. SHAO, AND \\ ANDREW R. WILKINSON \\ Neonatal Unit [Z.D.J., D.M.B., J.W., X.X., A.R.W.], Department of Paediatrics, University of Oxford, John \\ Radcliffe Hospital, Headington, Oxford OX3 9DU, United Kingdom, and Children's Hospital [Z.D.J., \\ J.W., X.X., G.Q.C., X.M.S.], Fudan University, Shanghai 200032, China
}

\begin{abstract}
Dynamic changes in electrophysiology of brainstem auditory neurons during the first month after birth were studied in 51 term infants after perinatal asphyxia using maximum length sequence brainstem auditory evoked responses. The responses were recorded on d 1, 3, 5, 7, 10, 15, and 30 after birth. On d 1, wave III and V latencies and all interpeak intervals increased significantly at all repetition rates of clicks used (91-910/s), especially the higher rates (ANOVA, $p<0.05-0.0001$ ). On d 3, all these latencies and intervals increased further and differed more significantly from the normal control subjects. Thereafter, the latencies and intervals decreased progressively. On $\mathrm{d} 7$, wave $\mathrm{V}$ latency and all intervals still differed significantly from the control subjects. These dynamic changes were more significant at higher rates of clicks than at lower rates. On d 10 and 15, all intervals decreased significantly. On d 30, all wave latencies decreased to the values in the normal control subjects on the same day. The intervals also approached normal values, although the III-V and I-V intervals still increased slightly. These results indicate that hypoxic-ischemic brain damage persists during the first week, with a peak on d 3, and recovers progressively
\end{abstract}

\section{ABSTRACT}

thereafter. By $1 \mathrm{mo}$, the damage has largely returned to normal. Maximum length sequence brainstem auditory evoked responses results correlated well with the stage of hypoxic-ischemic encephalopathy during the first week. The present study revealed a general time course of brainstem pathophysiology after asphyxia, although there were individual variations. Our findings can be used as a reference to monitor cerebral function and help judge the value of neuroprotective or therapeutic interventions. The first week, particularly the first $3 \mathrm{~d}$, is a critical period of hypoxic-ischemic brain damage, and early intervention may prevent or reduce deterioration of the damage. (Pediatr Res 54: 680-687, 2003)

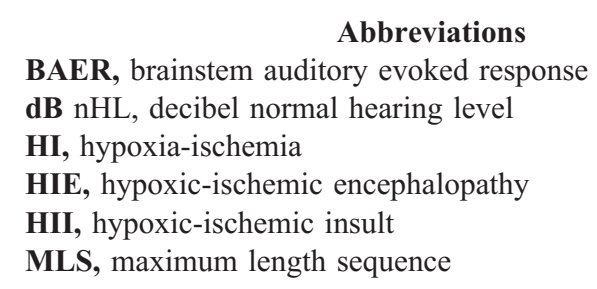

There is growing evidence to suggest that HII after asphyxia produces permanent brain damage by processes that continue for many hours after the insult (1-3). The neurotoxic cascade leads to delayed cell death hours, days, or months later. This may provide an opportunity for early therapy aimed at preventing further damage of the brain. However, further study of the pathophysiologic process of HI brain damage, especially dur-

Received September 27, 2002; accepted March 31, 2003.

Correspondence: Ze Dong Jiang, M.D., Neonatal Unit, Department of Paediatrics, University of Oxford, John Radcliffe Hospital, Headington, Oxford OX3 9DU, United Kingdom; e-mail: zedong.jiang@paediatrics.ox.ac.uk

Supported by the Wellcome Trust, R\&D Anglia and Oxford Regional Health Authority, Children Nationwide Medical Research Fund, and Defeating Deafness, UK.

DOI: 10.1203/01.PDR.0000084084.62571.12 ing the critical period, is of great importance for planning any intervention with neuroprotective and therapeutic measures $(4-8)$.

A major neuropathologic pattern of HIE in term infants is known to be selective neuronal necrosis. After perinatal asphyxia discrete lesions are very common in the brainstem (9-11). In acute total asphyxia there is extensive neuronal necrosis. The damage preferentially affects the brainstem, thalamus, and basal ganglia with the cerebral cortex being relatively spared (11). The lesions in the brainstem frequently involve the auditory pathway, including loss of neurons with gliosis or ischemic cell changes in the cochlear nuclei, superior olive, and inferior colliculus, suggesting that this pathway is particularly sensitive to HII $(9,10)$. Thus, study of this path- 
way can provide important information about cerebral function after asphyxia. A noninvasive and objective test that reflects functional status of this pathway is the BAER (12). This response is very sensitive to arterial blood oxygen levels and hypoxia $(13,14)$, and has been used to assess auditory function and, in a more general sense, cerebral function in infants after asphyxia (12, 15-20). However, the BAER, recorded using conventional averaging techniques, often fails to demonstrate early or subtle neural impairment.

Increasing the repetition rate of acoustic stimuli while recording the BAER may improve the detection of neural impairment $(12,15)$. Our recent studies indicate that to improve the detection of neuropathology the repetition rate of stimuli should be high enough to effectively stress the large number of neurons along the brainstem auditory pathway $(18,21,22)$. However, conventional averaging technique imposes a rate limit of approximately $100 / \mathrm{s}$, which restricts the ability of the method of increasing click rate to improve early detection of neural impairment $(23,24)$. We have recently studied a relatively new technique, the MLS BAER, in infants of various ages (23-25). The major advantage of this technique is that it can present acoustic stimuli at much higher rates (up to 1000/s or even higher) than is possible using conventional BAER (23-29). The higher rates provide a much greater temporal or physiologic challenge to brainstem auditory neurons, potentially enhancing the detection of neuropathology. We have found that this relatively new technique is a valuable method to detect early brain damage after perinatal asphyxia and is more sensitive than conventional $\operatorname{BAER}(24,25)$.

To date, limited information is available about the detailed time course of HI brain damage after asphyxia, revealed using noninvasive methods. We have recently studied dynamic changes in brainstem auditory electrophysiology in infants who suffered perinatal asphyxia by serially recording the MLS BAER during the first month after birth to understand the detailed pathophysiologic process of brain damage after asphyxia. Wave latencies and interpeak intervals in the MLS BAER were analyzed in detail to assess central auditory function. Because preterm asphyxiated infants often have other associated perinatal problems, which may mask the significance of intrapartum HI (30), we have confined this study to term infants. Subjects with peripheral auditory impairment were excluded to avoid the potential peripheral influence on central components of the MLS BAER.

\section{METHODS}

Subjects. Sixty-five newborn infants who suffered perinatal asphyxia, or HI (39 boys and 26 girls), were recruited either from the Neonatal Unit, Department of Paediatrics, John Radcliffe Hospital, University of Oxford, United Kingdom ( $n=$ 26), or from the Neonatal Unit, Children's Hospital of Fudan University, Shanghai, China $(n=39)$. Gestational age ranged between 37 and $42 \mathrm{wk}(39.3 \pm 1.5 \mathrm{wk})$, and birth weight between 2150 and $4550 \mathrm{~g}(3212 \pm 505 \mathrm{~g})$. These subjects were studied on d 1, 3, 5, 7, 10, 15, and 30 after birth.

The criteria for perinatal asphyxia were 1) clinical signs of HI (hypotonia with reduced or no spontaneous movements, increased threshold for primitive reflexes, lethargy or comatose, absence or very weak suck and requirement of tube feeds, or seizures), and other signs of hypoxia, including frequent depression and failure to breathe spontaneously at birth (30, 31); and 2) depression of the Apgar score ( $\leq 6$ at $5 \mathrm{~min}$ ). Most subjects had meconium staining of the amniotic fluid and umbilical cord blood $\mathrm{pH}<7.10$. Cranial computed tomography or ultrasound scan showed that 52 infants had HI. Those who had congenital malformation and congenital or perinatal infection of the CNS were excluded.

The normal control subjects were 38 healthy newborn infants (18 boys and 20 girls), recruited from the maternity words, Department of Obstetrics and Gynaecology, John Radcliffe Hospital, University of Oxford, United Kingdom. None had any major perinatal conditions or complications such as asphyxia, intrauterine growth retardation, intrauterine or postnatal infection, or congenital abnormality. Gestational age ranged between 37 and 41 wk ( $39.0 \pm 1.3$ wk). Apgar scores were all $\geq 8$ at both 1 and $5 \mathrm{~min}$. Monaural BAER thresholds, determined by conventional BAER at $21 \mathrm{clicks} / \mathrm{s}$, were all $<20$ $\mathrm{dB} \mathrm{nHL}$ at the time of testing. The MLS BAER was recorded on d 1-3 (first recording) and d 30 after birth (second recording).

Recording and analysis of the MLS BAER. The study protocols and procedures were approved by the Central Oxford Research Ethics Committee and the Children's Hospital Ethics Committee of Fudan University. Informed consent of parents and the pediatrician in charge was obtained.

A Bravo Portable Evoked Potential System (Nicolet Biomedical Inc, Madison, WI, U.S.A.) was used to record and analyze the MLS BAER. The procedures were basically the same as previously reported $(23,24)$. Briefly, the subjects lay supine in their crib. The recording started after the baby fell asleep naturally, often after a feeding. No sedatives were used. The left ear was tested in all subjects. Three gold-plated disk electrodes were placed at the middle forehead (positive), the ipsilateral earlobe (negative), and the contralateral earlobe (ground). Interelectrode impedances were maintained at $<10$ $\mathrm{k} \Omega$.

Rarefaction clicks of $100 \mu$ s were delivered monaurally through a TDH 39 earphone at repetition rates of 91/s, 227/s, $455 / \mathrm{s}$, and $910 / \mathrm{s}$, equivalent to a minimum interpulse interval (the duration of the sequence) of 11.1, 4.4, 2.2, and $1.1 \mathrm{~ms}$, respectively. The intensity of clicks was $60 \mathrm{~dB} \mathrm{nHL}$ for all infants. Higher intensities were also used in those with a BAER threshold $>20 \mathrm{~dB} \mathrm{nHL}$ so that the data of BAER central components in asphyxiated infants can be compared with the normal control subjects at the same hearing level (i.e. $\geq 40 \mathrm{~dB}$ above their thresholds). Conventional BAER was also recorded at $21 / \mathrm{s}$ for comparison and for the determination of BAER threshold (the lowest intensity at which wave $\mathrm{V}$ can be recognized).

The evoked brain responses were amplified and filtered at $100-3000 \mathrm{~Hz}$. If the data exceeded $91 \%$ of the sensitivity factor setting $(51 \mu \mathrm{V})$, that sweep (artifact) was automatically rejected by the system. Sampling was manually discontinued whenever there were excessive muscle artifacts on the monitoring oscilloscope. Brain responses to 1500 clicks were aver- 
aged for each run. A sweep duration of $24 \mathrm{~ms}$ was used in recording the MLS BAER, and a duration of $12 \mathrm{~ms}$ was used in conventional BAER. As shown in the sample recordings of the MLS BAER in asphyxiated infants in our previous paper (24), duplicate recordings were made in response to each stimulus condition to examine reproducibility. The clicks were presented in the sequence of $91 / \mathrm{s}, 227 / \mathrm{s}, 455 / \mathrm{s}$, and $910 / \mathrm{s}$ in the first run. A reverse sequence was used in the second run. Mean measurements of the two replicated recordings to each stimulus condition were used for data analyses.

Wave latencies and interpeak intervals in the MLS BAER were measured and analyzed in detail. All quantitative analyses of BAER variables were based on the data collected at the click intensity of $60 \mathrm{~dB} n H L$ for thresholds $\leq 20 \mathrm{~dB} n H L, 70 \mathrm{~dB}$ nHL for thresholds $>20-30 \mathrm{~dB} n H L$, or $80 \mathrm{~dB} \mathrm{nHL}$ for thresholds $>30-40 \mathrm{~dB}$ nHL. This allowed all data in the asphyxiated infants to be analyzed at a level $\geq 40 \mathrm{~dB}$ above the thresholds, the same as in the control subjects, and to be compared directly with those in the control subjects.

Because of a reduction of energy supply to the organ of Corti after asphyxia, cochlear function may be impaired in some cases. Furthermore, some subjects may have middle ear disorders during the course of study. It is known that peripheral hearing impairment affects the measurement of central BAER components, resulting in amplitude reduction and latency prolongation. If there is a high frequency hearing loss, I-V interpeak interval could be shortened because of a shift in the relative contributions of different regions of the cochlea to the click response, from the normally dominant high-frequency region $(2-4 \mathrm{kHz})$ to lower frequency regions of the cochlea associated with longer latencies. To minimize the potential effect of peripheral hearing impairment on MLS BAER central components, assessing central function more accurately, the data of 14 infants who had a BAER threshold $>25 \mathrm{~dB}$ nHL were excluded. Thus, the data presented in this report were collected from the remaining 51 children who had a BAER threshold $\leq 25 \mathrm{~dB}$ nHL.

Each asphyxiated infant was studied between two and five times, as appropriate. The number of infants on each study day (d 1, 3, 5, 7, 10, 15, and 30 after birth) ranged between 23 and 32. Mean and SD of each BAER variable at each stimulus condition in the asphyxiated infants were compared with the normal control subjects using one-way ANOVA. Correlation of MLS BAER results with the stage of HIE was analyzed with Pearson's test.

\section{RESULTS}

During the whole period of the first month after birth, all latencies and interpeak intervals in the asphyxiated infants changed linearly with the change in click rate, which was more significant than in the normal control subjects, suggesting an increased rate-dependent change. The later components of the MLS BAER, i.e. those with longer latency and reflecting more central function, changed more significantly than the earlier components, i.e. those with shorter latency and reflecting more peripheral function. Wave I latency, reflecting peripheral auditory function, lacked systematic changes, and did not show any consistent abnormalities at any day of recording (Fig. 1). By contrast, wave $\mathrm{V}$ latency and the interpeak intervals of $\mathrm{I}-\mathrm{V}$, I-III, and III-V, mainly reflecting central auditory function, often showed systematic changes with change in the day of recording and repetition rate of click stimuli, and were often abnormal, especially at higher rates of clicks (Figs. 1 and 2).

Day 1. The change in wave V latency with the increase in click rate, or rate-dependent change, in the asphyxiated infants
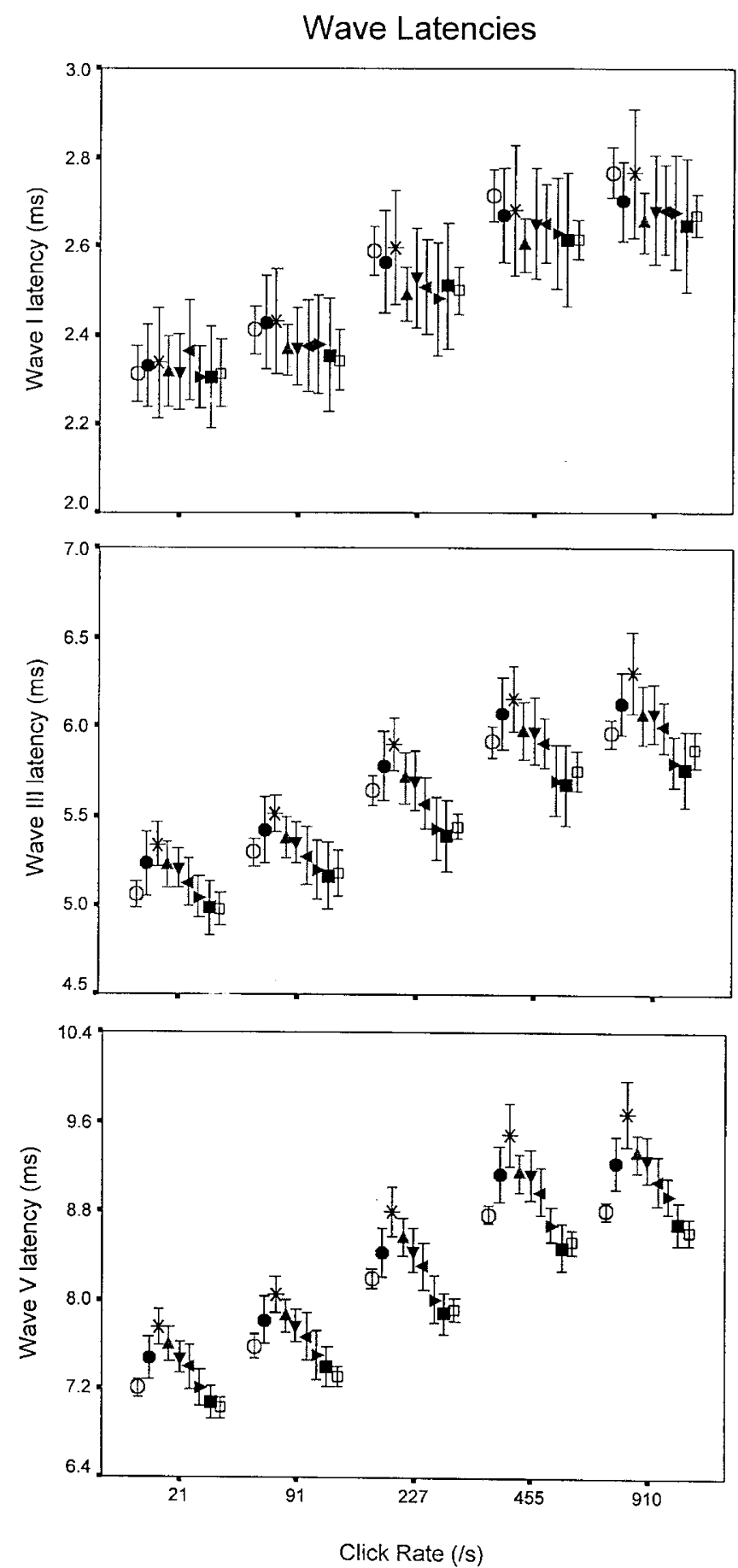

Figure 1. Mean and SEM of wave I, III, and V latencies at different repetition rates of click stimuli ( $\geq 40 \mathrm{~dB}$ above BAER threshold) during the first month after birth. The symbols in sequence stand for the data of normal control infants at term, asphyxiated infants on $\mathrm{d} 1,3,5,7,10,15$, and 30 , and the control subjects on $\mathrm{d} 30$. 
Interpeak Intervals
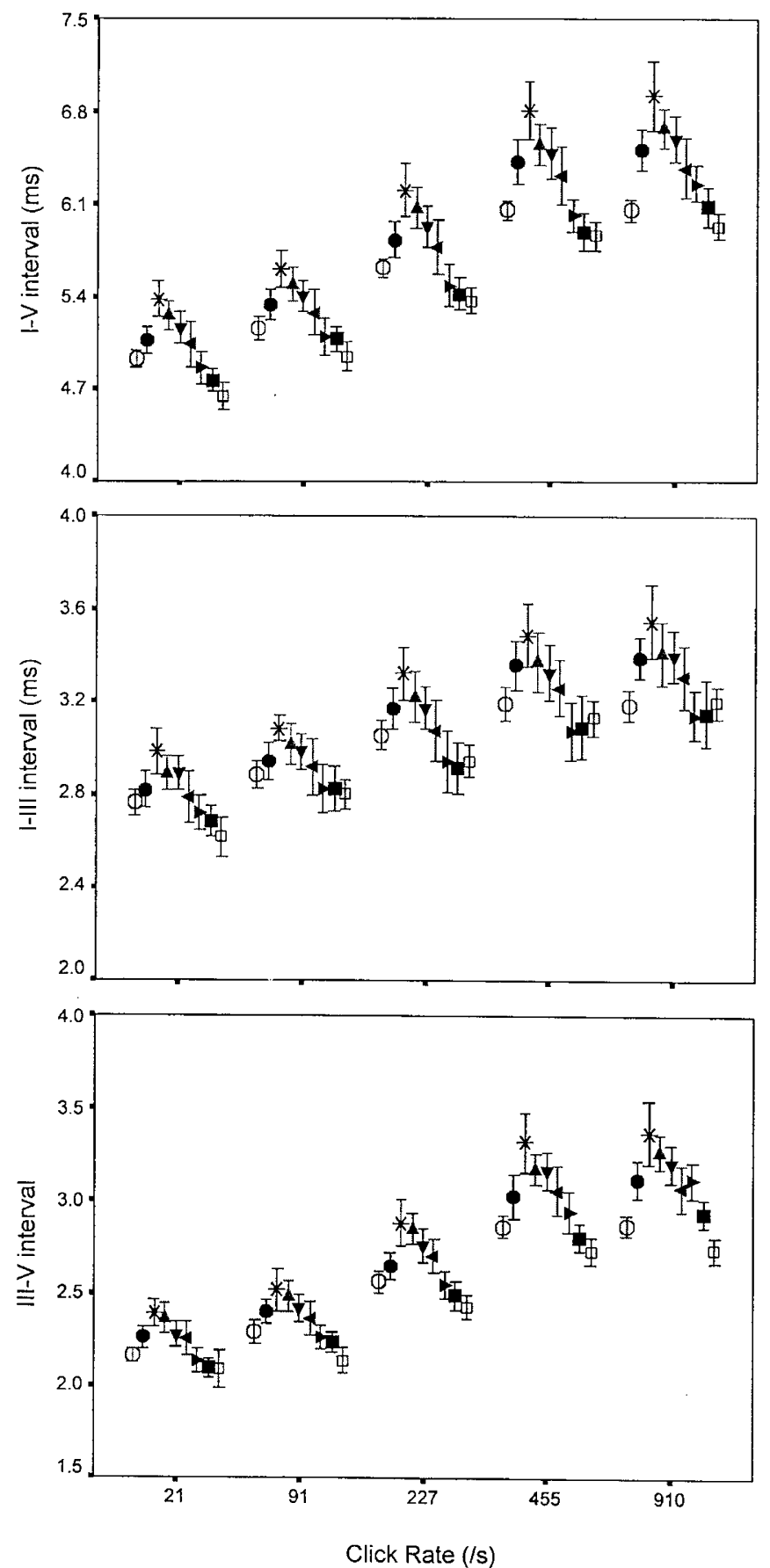

Figure 2. Mean and SEM of I-V, I-III, and III-V intervals at different rates of clicks ( $\geq 40 \mathrm{~dB}$ above BAER threshold) during the first month after birth. The symbols in sequence stand for the data of normal control subjects at term, asphyxiated infants on $\mathrm{d} 1,3,5,7,10,15$, and 30 , and the control subjects on d 30 .

was significantly greater than in the control subjects at term. The latency increased significantly at all rates of clicks, especially at 455/s and 910/s (ANOVA, $p<0.01-0.001$; Fig. 1). By contrast, wave I latency did not differ significantly from that in the control subjects at any rates (Fig. 1). Wave III latency increased slightly at all rates used (all $p<0.05$; Fig. 1).
All intervals of $\mathrm{I}-\mathrm{V}, \mathrm{I}-\mathrm{III}$, and III-V in the asphyxiated infants increased significantly (ANOVA, $p<0.05-0.0001$; Fig. 2). The differences in these intervals between the asphyxiated infants and the normal control subjects at term increased with the increase in the rate of clicks, i.e. the rate-dependent changes increased in the asphyxiated infants. The III-V/I-III interval ratio did not differ significantly from the control subjects at any rate (Fig. 3).

Day 3. Wave V latency in the asphyxiated infants increased further and differed significantly from that on $\mathrm{d} 1$ at all rates used (ANOVA, $p<0.05-0.01$; Fig. 1). The difference in the latency between the asphyxiated infants and the control subjects at term was more significant than on $\mathrm{d} 1$ at all rates $(p<$ 0.001-0.0001). Wave III latency also increased slightly further and was significantly longer than in the control subjects at term at all rates used ( $p<0.01-0.001 ;$ Fig. 1$)$. Wave I latency did not show any significant changes in comparison with that on $\mathrm{d}$ 1 (Fig. 1).

All the I-V, I-III, and III-V intervals in the asphyxiated infants increased further, and differed significantly from those on $\mathrm{d} 1$ at all rates of clicks (ANOVA, $p<0.05-0.001$; Fig. 2). The differences in these intervals between the asphyxiated infants and the control subjects at term increased with the increase in click rate and were more significant than on $\mathrm{d} 1(p$ $<0.001-0.0001)$. The III-V/I-III interval ratio tended to increase slightly at higher rates in comparison with that on $\mathrm{d} 1$, suggesting that the III-V interval increased slightly more than the I-III interval at high-rate stimulation (Fig. 3).

Day 5. All wave latencies in the asphyxiated infants tended to decrease in comparison with those on $\mathrm{d} 3$ (Fig. 1). Wave $\mathrm{V}$ latency decreased significantly at all rates (ANOVA, all $p<$ $0.05-0.01$ ), and was similar to the values on $\mathrm{d} 1$. Nevertheless, the latency remained significantly longer than in the control subjects at term at all rates $(p<0.01-0.0001)$. Wave III

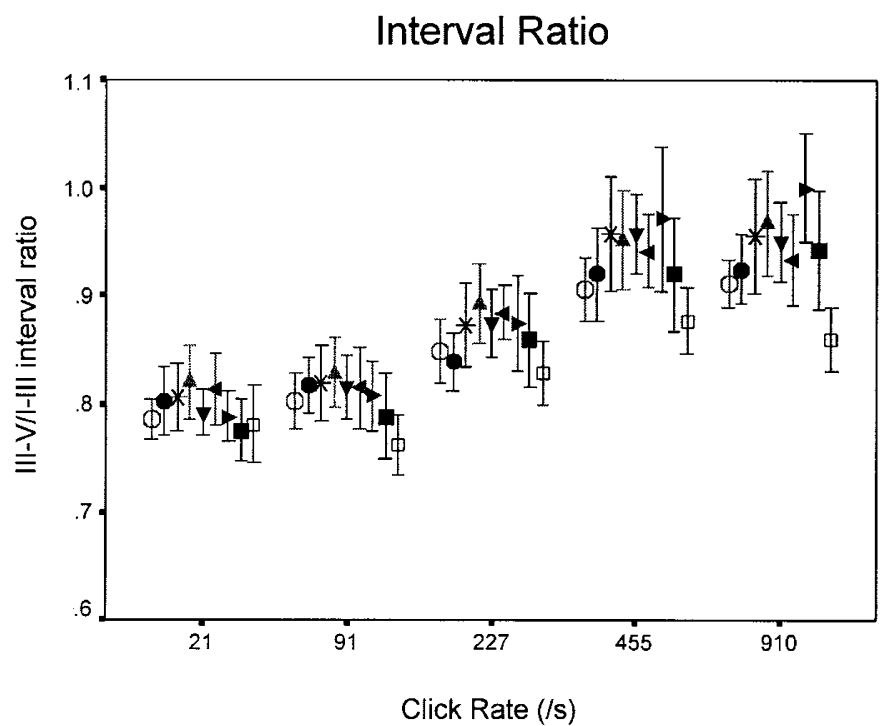

Figure 3. Mean and SEM of III-V/I-III interval ratio at different rates of clicks ( $\geq 40 \mathrm{~dB}$ above BAER threshold) during the first month after birth. The symbols in sequence stand for the data of normal control subjects at term, asphyxiated infants on $\mathrm{d} 1,3,5,7,10,15$, and 30 , and the control subjects on d 30 . 
latency also decreased at all rates of clicks in comparison with that on $\mathrm{d} 3$ (ANOVA, all $p<0.05$ ). The latency was similar to the values on $\mathrm{d} 1$, but tended to be slightly longer than in the control subjects at term. Wave I latency tended to decrease slightly at higher rates (Fig. 1).

Compared with those on d 3, all interpeak intervals in the asphyxiated infants tended to decrease (Fig. 2). The I-V and I-III intervals decreased significantly at all rates of clicks (ANOVA, $p<0.05-0.01$ ). The III-V interval also decreased significantly at 455/s and 910/s (both $p<0.05$ ). All these intervals in the asphyxiated infants remained significantly longer than in the control subjects at term $(p<0.01-0.0001)$. The III-V/I-III interval ratio did not show any significant changes and was similar to the values at different rates on $\mathrm{d} 3$ (Fig. 3).

Day 7. No significant further changes were found in any wave latencies at any rates of clicks (Fig. 1). Similarly, the III-V/I-III interval ratio did not show any significant change (Fig. 3). All interpeak intervals tended to decrease slightly (Fig. 2).

Compared with those in the control subjects at term, the I-V and III-V intervals still increased significantly, especially at higher rates $(p<0.01-0.0001)$, although the I-III interval increased less significantly $(p<0.05-0.01)$. Wave $\mathrm{V}$ latency also still increased $(p<0.01-0.001)$.

Day 10 and later. After the first week, wave III and V latencies and all intervals in the asphyxiated infants recovered progressively and significantly, although wave I latency did not show any significant changes or abnormalities (Figs. 1 and 2).

On d 10 and 15, wave III latency almost recovered, but wave V still increased slightly. On d 30, all latencies of waves I, III, and $\mathrm{V}$ reached the values in the control subjects on the same day (Fig. 1). There were no differences in rate-dependent changes in the wave latencies between the asphyxiated infants and the control subjects on $\mathrm{d} 30$.

On d 10, the I-III interval already approached the values in the control subjects at term at most rates used, except at the highest rate, 910/s ( $p<0.05$; Fig. 2). The I-V interval and the III-V intervals were still significantly longer than in the control subjects at term at all rates, particularly at higher rates (ANOVA, $p<0.05-0.001$ ). On d 15, all intervals continued to decrease.

On d 30, both the I-V and III-V intervals decreased further, but still tended to increase in comparison with the control subjects on the same day $(p<0.05-0.01$ for $\mathrm{I}-\mathrm{V}$ at $21 / \mathrm{s}, 91 / \mathrm{s}$, and $910 / \mathrm{s}$ clicks, and $p<0.05-0.001$ for III-V at $91-910 / \mathrm{s}$; Fig. 2). The rate-dependent changes in the two intervals did not differ from the control subjects. The I-III interval did not differ significantly from the control subjects at any rates. The III-V/ I-III interval ratio still tended to increase at higher rates $(p<$ 0.5 at $455 / \mathrm{s}$ and $p<0.01$ at $910 / \mathrm{s}$; Fig. 3), resulting mainly from the increase in the III-V interval and to a lesser extent from the slightly decrease in the I-III interval.

Correlation between MLS BAER results and the stage of HIE. In our cohort, there were 11 subjects with mild asphyxia, 28 moderate, and 12 severe, according to the grading system of Sarnat and Sarnat $(31,32)$. Analysis of the correlation showed that wave $\mathrm{V}$ latency correlated significantly with the stage of
HIE at all rates of clicks on d 1, 3, 5, and $7\left(r^{2}=0.32-0.59\right.$, $p<0.05-0.01$ ), except at $21 / \mathrm{s}$ and $91 / \mathrm{s}$ on $\mathrm{d} 5$. The correlation tended to be more significant at higher rates. After the first week, there was no significant correlation at any rate on any day. No significant correlation was found between wave I and III latencies and the stage of HIE on any day during the first month. When the data on $\mathrm{d} 1$ and 3 were pooled together, wave III latency showed significant correlation with the stage of HIE.

The I-V interval in the asphyxiated infants correlated significantly with the stage of HIE at all rates of clicks on d 1, 3, 5 , and 7, particularly at higher rates $\left(r^{2}=0.38-0.66, p<\right.$ $0.05-0.01)$. Thereafter, there was no significant correlation at any day, except at $227 / \mathrm{s}$ clicks on d $10\left(r^{2}=0.42, p<0.05\right)$. The I-III and III-V intervals correlated significantly with the stage of HIE at all rates of clicks on d 1, 3, 5, and $7\left(r^{2}=\right.$ $0.38-0.66, p<0.05-0.01$ ), except at $21 / \mathrm{s}$ clicks on $\mathrm{d} 1$ for the III-V interval. After the first week, significant correlation was found only at 227-910/s clicks on d 10 for the I-III interval $\left(r^{2}\right.$ $=0.32-0.39, p<0.05)$ and at $227 / \mathrm{s}$ on $\mathrm{d} 10$ for the III-V interval $\left(r^{2}=0.41, p<0.05\right)$. The III-V/I-III interval ratio did not correlate with the stage of HIE at any rate on any day.

\section{DISCUSSION}

Time course of HI brain damage in asphyxiated infants. The present study revealed a general time course of brainstem pathophysiology after perinatal asphyxia. Our findings suggest that after perinatal asphyxia HI brain damage persists during the first week after birth, with a peak at approximately $\mathrm{d} 3$ and a tendency to recover progressively thereafter. It appears that the first week, particularly the first $3 \mathrm{~d}$, is a critical period of $\mathrm{HI}$ brain damage. By $1 \mathrm{mo}$, the damage has largely returned to normal.

Pathophysiologic process of auditory impairment during the critical period of HI brain damage. The increase in BAER intervals after asphyxia reflects an impaired neural conduction and synaptic transmission of the brainstem after HI brain damage. Our results suggest that perinatal asphyxia has a major effect on central auditory function during the first week after birth. In the first day, our major findings in the asphyxiated infants, i.e. a significant increase in wave $\mathrm{V}$ latency and the I-V, I-III, and III-V intervals, reflect acute HI damage to the central nervous system, resulting in impaired neural conduction. The further increase in wave III and V latencies and all intervals on $\mathrm{d} 3$ indicates a further increase in central conduction. Apparently, the pathology of HI brain damage progresses during the first $3 \mathrm{~d}$. This finding is in accordance with the clinical observation that in some cases the effect of perinatal asphyxia becomes manifest only some days after resuscitation.

The rate-dependent changes in the BAER primarily reflect neural processes concerning the efficacy of central synaptic transmission, as well as neural synchronization and metabolic status of auditory neurons in the brainstem after the presentation of a physiologic challenge (24). Asphyxia disturbs the metabolism of neurons and depresses the electrophysiologic function of synapses that transmit developmental as well as regulatory signals between neurons. Synaptic efficacy is related 
to the mechanisms for synthesis, release, and uptake of neurotransmitters. Significant HI causes excessive release of glutamate from the presynaptic transmitter site and compromises the uptake pumps of glutamate within the synaptic cleft. As a result, the concentration of glutamate increases dramatically within minutes, leading to an impairment of synaptic transmission and irreversible neuronal injury.

In the present study the rate-dependent changes in the MLS BAER in the asphyxiated infants were more significant than in the control subjects. MLS BAER abnormalities increase with the increase in click rate. The dynamic changes in the MLS BAER were more significant at higher rates of clicks than at lower rates. These findings reflect a decreased efficacy of central synaptic transmission or a decreased ability of central neurons to recover in time to transmit the next stimulus-evoked response after $\mathrm{HI}$. The finding that there was a further increase in the rate-dependent changes in wave latencies and interpeak intervals on $\mathrm{d} 3$ suggests that the impairment of central synaptic efficacy deteriorates during the first $3 \mathrm{~d}$.

After the first $3 \mathrm{~d} \mathrm{HI}$ brain damage tends toward recovery. However, on $\mathrm{d} 7$ the central components of the MLS BAER remained significantly abnormal, especially at higher rates. This finding suggests that central conduction and synaptic efficacy in the auditory brainstem remain far from normal, and that HI brain damage persists during the first week.

The III-V/I-III interval ratio tended to increase in the MLS BAER during the period of study, although it did not show any significant abnormalities in conventional BAER. This increase suggests that $\mathrm{HI}$ damage to the more central part of the auditory brainstem is relatively more significant than to the more peripheral part of the brainstem.

Recovery process of auditory impairment after the critical period of HI brain damage. After the first week the HI damage to central auditory neurons recovered significantly and progressively. The recovery process continues up to the end of the first month studied. By then, the impaired neural conduction in the brainstem, reflected by the increased wave $\mathrm{V}$ latency and interpeak intervals, has largely returned to normal. The finding that at higher rates the I-III interval decreased slightly more than the III-V interval suggests that the more peripheral part of the brainstem auditory pathway tends to recover sooner than the more central part of the auditory brainstem.

After the critical period of $\mathrm{HI}$ brain damage during the first week, the increased rate-dependent changes in wave latencies and interpeak intervals decreased progressively, and returned to normal on $\mathrm{d} 30$. Thus, by 1 mo the impaired central synaptic efficacy has almost recovered.

The data presented in this report show a general trend of the changes in the MLS BAER after perinatal asphyxia. Nevertheless, there were individual variations. Some subjects, mainly those after mild asphyxia, recovered sooner (d 3 or 5 ) than others, mainly those after severe asphyxia. In fewer cases, the intervals in the MLS BAER increased further some days after birth. This is consistent with our clinical observations in these subjects that the symptoms of HIE become more significant some days or even 1 wk after birth.

We found that BAER abnormalities in the asphyxiated infants became more significant as the rate of clicks was increased. The dynamic changes in the BAER were more dramatic at higher rates. Therefore, the high rates provided by the MLS technique can improve the diagnostic value of the BAER for HI brain damage.

In addition, we carried out a preliminary observation of the prognostic value of MLS BAER testing in some of the subjects. The infants whose MLS BAER recovered sooner, e.g. on d 3 or 5, often had favorable outcome whereas those who had persistent MLS BAER abnormalities tended to have poorer outcome. We also found that the MLS BAER had a better prognostic value than conventional BAER. However, neurodevelopment has not been assessed in most of our subjects, and the number of subjects at each stage of HIE was relatively small at present. More subjects are needed before any detailed statistical analysis can be performed to elucidate the relationship between MLS BAER changes and the stage of HIE and to determine the prognostic value of the MLS BAER.

Implications for dynamic changes in brainstem auditory electrophysiology after perinatal asphyxia. The present study found a good correlation of wave V latency and the I-V, I-III, and III-V intervals with the stage of HIE, particularly at higher rates of clicks, during the first week after birth. From d 10 to d 30, MLS BAER variables did not correlate significantly with the stage of HIE. A likely interpretation of this is that after the first week MLS BAER abnormalities have significantly recovered and the differences in the MLS BAER between different stages of HIE have become very small. Wave V latency and the $\mathrm{I}-\mathrm{V}, \mathrm{I}-\mathrm{III}$, and III-V intervals are MLS BAER variables that mainly reflect central neural function. The significant correlation of these variables with the stage of HIE suggest that these variables are particularly useful to monitor pathophysiologic changes in the brain after perinatal asphyxia and the MLS BAER can provide useful information for the management of infants after perinatal asphyxia.

Our findings of dynamic changes in the MLS BAER in infants after perinatal asphyxia provide noninvasive electrophysiologic evidence of pathophysiologic process of HI brain damage during the first month after asphyxia. The deterioration of the damage on $\mathrm{d} 3$ supports the notion of secondary energy failure and secondary cerebral hypoperfusion. It also suggests that there is a delayed neuronal impairment after HII and intervention during the first hours after birth may prevent or reduce the deterioration.

There is a growing consensus that not only do some cells die during HI, but many more may die hours or days later (2-4). A magnetic resonance spectroscopy study in infants after asphyxia showed that cerebral energy metabolism was usually normal soon after resuscitation, but oxidative phosphorylation became impaired 9 to $24 \mathrm{~h}$ later and remained low for many days, suggesting a delayed impairment of energy metabolism (33). The death of neurons in the postasphyxial period may be initiated by necrosis as the direct result of the HII, or occur as the result of apoptosis. Secondary neuronal death probably occurs as a combination of the two interrelated processes (30). The mechanisms of this delayed cell death are complex. It has been recognized that apoptosis is an important aspect of HI brain damage, and may play an important role in delayed cell death. 
Intervention with neuroprotective and therapeutic measures is crucial for improving the outcome of asphyxiated infants. The delayed cell death or deteriorating neural function shortly after birth provides an opportunity for therapy aimed at preventing further brain damage. It may be possible to intervene before delayed cell death occurs and so reduce brain damage. Experiments in animals have shown that a large number of treatments would reduce brain injury if given soon after the insult (1). Recently, moderate hypothermia to protect against brain injury has undergone vigorous investigation by many researchers around the world $(2,4,6,7,31)$. Cooling the brain to approximately $32^{\circ} \mathrm{C}$ is one of several interventions that can be applied after HI to modify the process of brain cell death and so reduce brain damage. Apparently, rescue treatment of $\mathrm{HI}$ after resuscitation appears to be a real possibility. The present study provides baseline data for dynamic changes in brainstem electrophysiology after perinatal asphyxia. These can be used as reference data to monitor cerebral function after asphyxia and to assess the responses of the brain to neuroprotective or therapeutic interventions or help judge the value of the interventions. The $\mathrm{I}-\mathrm{V}$ and $\mathrm{III}-\mathrm{V}$ intervals can be used as major MLS BAER variables. The significant correlation of MLS BAER results with the stage of HIE suggests that the MLS BAER may also be useful in assisting selection of the appropriate infants to receive early interventions aimed at improving the outcome. The infant who shows significant abnormalities in the MLS BAER may be a suitable candidate for early interventions, e.g. hypothermia.

Recent studies in animals with experimental neonatal HI have shown that the BAER correlated to the extent of HII and that the BAER is useful in assessing the neuroprotective effects of hypothermia on neonatal HII $(34,35)$. The authors indicated that the BAER provides a stable and objective measure to assess cerebral function after neonatal HII. Similar to our previous report (24), the present study shows that the MLS BAER can improve the value of conventional BAER in the assessment of cerebral function in infants after perinatal asphyxia. It is obviously possible that this technique can potentially improve the value of the BAER in the assessment of treatment effects, e.g. neuroprotective effects of hypothermia.

A time frame in which recording of the MLS BAER is most valuable for clinic application, particularly to the infant who suffers severe, prolonged asphyxia, can be selected from the dynamic changes in the MLS BAER. The first recording can be made in the first day after birth for the early detection of HI brain damage and for assessment of the severity of the damage. The second recording can be made at approximately d 3 to examine whether the damage has deteriorated or not. The third recording can be made on d 7-10 to assess any significant recovery. The fourth recording can be made at 1 mo to examine whether the damage has largely returned to normal or not.

\section{CONCLUSIONS}

In conclusion, the present study revealed a general trend of electrophysiologic changes in the brainstem of neonates after asphyxia. Our findings, which have never been reported before, not only provide new insights into the pathophysiologic pro- cess of perinatal HI brain damage, but also may have important implications for the improvement of management of asphyxiated infants and for studying neuroprotective or therapeutic interventions.

Acknowledgments. The authors thank the doctors and nurses at the Neonatal Unit of the Department of Paediatrics, John Radcliffe Hospital, University of Oxford and in the Children's Hospital, Fudan University, Shanghai for their enthusiastic support and assistance in the recruitment of subjects and collecting data.

\section{REFERENCES}

1. Johnston M, Trescher WH, Ishida A, Nakajima W 2001 Neurobiology of hypoxicischemic injury in the developing brain. Pediatr Res 49:735-741

2. Gunn AJ, Gunn TR, de Haan HH, Williams CE, Gluckman PD 1997 Dramatic neuronal rescue with prolonged selective head cooling after ischemia in fetal lambs. J Clin Invest 99:248-256

3. Kirino T 1997 Delayed neuronal death in the gerbil hippocampus following ischaemia. Brain Res 239:57-69

4. Taylor D, Mehmet H, Cady EB, Edwards AD 2002 Improved neuroprotection with hypothermia delayed by 6 hours following cerebral hypoxia-ischemia in the 14-dayold rat. Pediatr Res 51:13-19

5. Groenendaal F, de Vries LS 2000 Selection of babies for intervention after birth asphyxia. Semin Neonatol 5:17-32

6. Azzopardi D, Robertson NJ, Cowan FM, Rutherford MA, Rampling M, Edwards AD 2000 Pilot study of treatment with whole body hypothermia for neonatal encephalopathy. Pediatrics 106:684-694

7. Whitelaw A 2000 Systematic review of therapy after hypoxic-ischaemic brain injury in the perinatal period. Semin Neonatol 5:33-40

8. Wyatt JS, Thoresen M 1997 Hypothermia treatment and the newborn. Pediatrics 100:1028-1030

9. Dambska M, Laure-Kamionowska M, Liebhart M 1987 Brainstem lesions in the course of chronic fetal asphyxia. Clin Neuropathol 6:110-115

10. Leech RW, Alvord Jr EC 1977 Anoxic-ischemic encephalopathy in the human neonatal period: the significance of brain stem involvement. Arch Neurol 34:109-113

11. Pasternak JF 1993 Hypoxic-ischemic brain damage in the term infants: lessons from the laboratory. Pediatr Clin North Am 40:1061-1072

12. Chiappa KH 1990 Brainstem auditory evoked potentials: methodology. In: Chiappa $\mathrm{KH}$ (ed) Evoked Potentials in Clinical Medicine. Raven Press, New York, pp 173-221

13. Inagaki M, Kaga M, Isumi H, Hirano S, Takashima S, Nanba E 1997 Hypoxiainduced ABR changes and heat shock protein expression in the pontine auditory pathway of young rabbits. Brain Res 757:111-118

14. Sohmer H, Freeman S, Gafni M, Goitein K 1986 The depression of the auditory nerve-brain-stem evoked response in hypoxemia: mechanism and site of effect. Electroencephalogr Clin Neurophysiol 64:334-338

15. Hecox K, Cone B, Blaw M 1981 Brainstem auditory evoked response in the diagnosis of pediatric neurologic diseases. Neurology 31:832-839

16. Jiang ZD, Tierney TS 1996 Long-term effect of perinatal and postnatal asphyxia on developing human auditory brainstem responses: brainstem impairment. Int J Pediatr Otorhinolaryngol 34:111-127

17. Jiang ZD 1998 Maturation of peripheral and brainstem auditory function in the first year following perinatal asphyxia-a longitudinal study. J Speech Lang Hear Res 41:83-93

18. Jiang ZD, Brosi DM, Wilkinson AR 2001 Comparison of brainstem auditory evoked responses recorded at different presentation rates of clicks in neonates after asphyxia. Acta Paediatr 90:1416-1420

19. Karmel BZ, Gardner JM, Zappulla RA, Magnano CL, Brown EG 1988 Brainstem auditory evoked responses as indicators of early brain insult. Electroencephalogr Clin Neurophysiol 71:429-442

20. Majnemer A, Rosenblatt B, Riley P 1988 Prognostic significance of the auditory brainstem evoked response in high-risk neonates. Dev Med Child Neurol 30:43-52

21. Jiang ZD 1999 Outcome of brainstem auditory electrophysiology in children who survived purulent meningitis. Ann Otol Rhinol Laryngol 108:429-434

22. Jiang ZD, Brosi DM, Wilkinson AR2002 Auditory neural responses to click stimuli of different rates in the brainstem of very preterm babies at term. Pediatr Res 51:454-459

23. Jiang ZD, Brosi DM, Wilkinson AR 1999 Neonatal brainstem auditory evoked response recorded using maximum length sequences. Biol Neonate 76:193-199

24. Jiang ZD, Brosi DM, Shao XM, Wilkinson AR 2000 Maximum length sequence brainstem auditory evoked response in infants after perinatal hypoxia-ischaemia. Pediatr Res 48:639-645

25. Jiang ZD, Brosi DM, Wang J, Shao XM, Wilkinson AR2002 Brainstem auditory electrophysiology following perinatal asphyxia revealed by MLS BER: a comparison between term and preterm infants. Pediatr Res 51:451A(abstr)

26. Burkard R, McGee J, Walsh EJ 1996 Effects of stimulus rate on the feline brain-stem auditory evoked response during development. I. Peak latencies. J Acoust Soc Am 100:978-990

27. Eysholdt U, Schreiner C 1982 Maximum length sequences - a fast method for measuring brainstem evoked responses. Audiology 21:242-250 
28. Lasky RE 1997 Rate and adaptation effects on the auditory evoked brainstem response in human newborns and adults. Hear Res 111:165-176

29. Lasky RE, Shi Y, Hecox KE 1994 Binaural maximum length sequence auditoryevoked brain-stem responses in human adults. J Acoust Soc Am 93:2077-2087

30. Evans DJ, Levene MI 1999 Hypoxic-ischaemic injury. In: Rennie JM, Roberton NRC (eds) Textbook of Neonatology. Churchill-Livingstone, Edinburgh, pp 1231-1251

31. Levene MI 2001 The asphyxiated newborn infant. In: Levene MI, Chervenak FA, Whittle M (eds) Fetal and Neonatal Neurology and Neurosurgery, 4th ed. ChurchillLivingstone, Edinburgh, pp 471-504

32. Sarnat HB, Sarnat MS 1976 Neonatal encephalopathy following fetal distress. Arch Neurol 33:697-705
33. Wyatt JS, Edwards AD, Azzopardi D, Reynolds EO 1989 Magnetic resonance and near infrared spectroscopy for investigation of perinatal hypoxic-ischaemic brain injury. Arch Dis Child 64:953-963

34. Tomimatsu T, Fukuda H, Endoh M, Mu J, Watanabe N, Kohzuki M, Fujii E, Kanzaki T, Oxhima K, Doi K, Kubo T, Murata Y 2002 Effects of neonatal hypoxic-ischemic brain injury on skilled motor tasks and brainstem function in adult rats. Brain Res 926:108-117

35. Tomimatsu T, Fukuda H, Endoh M, Mu J, Kanagawa T, Hosono T, Kanzaki T, Doi K, Kubo T, Murata Y 2003 Long-term neuroprotective effects of hypothermia on neonatal hypoxic-ischemic brain injury in rats, assessed by auditory brainstem response. Pediatr Res 53:57-61 